\title{
Os impactos da Pandemia do COVID-19 na saúde mental dos estudantes
}

\author{
The impacts of the COVID-19 pandemic on the mental health of students \\ Los impactos de la pandemia COVID-19 en la salud mental de los estudiantes
}

Recebido: 15/07/2021 | Revisado: 20/07/2021 | Aceito: 23/07/2021 | Publicado: 31/07/2021

\author{
Gabrielly Maria Mendes de Barros \\ ORCID: https://orcid.org/0000-0002-7006-7484 \\ Faculdade de Ciências Médicas da Paraíba, Brasil \\ E-mail: gabriellymbarros@gmail.com \\ Filipe Carlos Eudes Pinto Valério \\ ORCID: https://orcid.org/0000-0002-2510-2172 \\ Faculdade de Ciências Médicas da Paraíba, Brasil \\ E-mail: filipecarloseudespinto@gmail.com \\ Maria Helena Franklin Domingos da Silva \\ ORCID: https://orcid.org/0000-0003-1775-7723 \\ Faculdades de Ciências Médicas da Paraíba, Brasil \\ E-mail: mariahelenafd@yahoo.com \\ Domennica Gomes Pecorelli \\ ORCID: https://orcid.org/0000-0002-0511-394X \\ Faculdade de Ciências Médicas da Paraíba, Brasil \\ E-mail: domennicagp@gmail.com \\ Vinicius Urquiza da Nóbrega Porto \\ ORCID: https://orcid.org/0000-0001-7734-8003 \\ Faculdade de Ciências Médicas da Paraíba, Brasil \\ E-mail: urquizavinicius2002@hotmail.com \\ Luisiane de Avila Silva \\ ORCID: https://orcid.org/0000-0003-2991-8918 \\ Faculdade de Ciências Médicas da Paraíba, Brasil \\ E-mail: luisianeavila@gmail.com
}

\begin{abstract}
Resumo
Objetivo: Demonstrar as estatísticas relacionadas aos impactos da pandemia na saúde mental dos estudantes. Métodos: Trata-se de uma revisão integrativa realizada em julho de 2021, nas plataformas LILACS, SciELO e Pubmed via MEDLINE. Foram selecionados artigos publicados no ano de 2020 e 2021, nos idiomas português, inglês, espanhol, francês, excluindo-se teses, dissertações, editorial, carta ao editor, opiniões e revisões de literatura. Resultados: Foram encontrados 202 artigos e após avaliação criteriosa 10 artigos foram selecionados sendo possível evidenciar o aumento de manifestações psicológicas negativas nos estudantes de ensino superior durante a pandemia da COVID-19. Considerações finais: Constatou-se que a maioria dos estudantes do ensino superior podem apresentar relatos de doenças mentais que podem estar relacionadas à falta de interação social diante do distanciamento obrigatório. Perante o exposto, este estudo buscou contribuir para reconhecimento de dados e para em breve sirva em prol as organizações de saúde com providências e soluções para a diminuição de casos de estudantes universitários com problemas mentais. Palavras-chave: Estudantes; COVID-19; Saúde dos estudantes; Saúde mental.
\end{abstract}

\begin{abstract}
Aim: To demonstrate statistics related to the impacts of the pandemic on students' mental health. Methods: This is an integrative review carried out in July 2021, on LILACS, SciELO and Pubmed platforms via MEDLINE. Articles published in 2020 and 2021 were selected, in Portuguese, English, Spanish, French, and excluded theses, dissertations, editorial, letter to the editor, opinions and literature reviews. Results: It was found 202 articles and after the appraisal it was selected 10 articles, it was possible to evidence the increase of negative psychological manifestations in higher education students during the COVID-19 pandemic. Final considerations: It was found that most higher education students may report mental illnesses that may be related to the lack of social interaction in the face of social distance. Given the above, this study sought to contribute to data recognition and to soon serve health organizations with measures and solutions to reduce cases of university students with mental problems.
\end{abstract}

Keywords: Students; COVID-19; Student health; Mental health.

\section{Resumen}

Objetivo: Demostrar estadísticas relacionadas con los impactos de la pandemia en la salud mental de los estudiantes. Metodos: Se trata de una revisión integradora realizada en julio de 2021, en plataformas LILACS, SciELO y Pubmed a través de MEDLINE. Se seleccionaron artículos publicados en 2020 y 2021, en portugués, inglés, español, francés, excluyendo tesis, disertaciones, editorial, carta al editor, opiniones y revisiones de literatura. Resultados:,Se encontraron 
202 artículos y después de una cuidadosa evaluación se seleccionaron 10 artículos, se pudo evidenciar el incremento de manifestaciones psicológicas negativas en estudiantes de educación superior durante la pandemia COVID-19. Consideraciones finales: Se encontró que la mayoría de los estudiantes de educación superior pueden reportar enfermedades mentales que pueden estar relacionadas con la falta de interacción social frente a la distancia obligatoria. Dado lo anterior, este estudio buscó contribuir al reconocimiento de datos y pronto servir a las organizaciones de salud con medidas y soluciones para reducir los casos de estudiantes universitarios con problemas mentales.

Palabras clave: Estudiantes; COVID-19; Salud del estudiante; Salud mental.

\section{Introdução}

A definição de saúde mental não é dada como um consenso, visto que, quando estudada, o diagnóstico pode ou não ser preciso. Os pacientes que sofrem com alterações na saúde mental muitas vezes se recusam a aceitar o diagnóstico por ideias anteriores em que os pacientes eram colocados em espaços sozinhos, distantes da sociedade (Gama, Campos \& Ferrer, 2014).

A entrada no ensino superior é um momento de diversas mudanças na vida dos estudantes, que, muitas vezes, estão apenas adaptados ao ritmo solicitado pelo ensino médio com pessoas anteriormente conhecidas em uma rotina também conhecida. Quando os estudantes ingressam no ensino superior é comum apresentarem angústias e medos relacionados ao seu desenvolvimento nessa nova realidade (Castro, 2017).

Durante o ano de 2020, os estudantes sofreram, além da dificuldade com a nova realidade universitária, a adaptação com o novo mundo de estudos em plataformas virtuais em virtude da pandemia no qual o mundo foi afetado (Santos, 2020).

A pandemia do novo coronavírus (SARS-Cov-2) teve início em 2019 na cidade de Wuhan (China), e foi reconhecida pela Organização Mundial da Saúde (OMS) em 11 de março de 2020 como uma emergência de saúde pública, levando a uma reorganização mundial em termos de saúde, segurança e tecnologia. Fez-se necessária a implantação do distanciamento social com isolamento de vulneráveis, o qual resultou, dentre tantos impactos nas mais diferentes esferas, no aumento do sofrimento mental nos estudantes, manifesto pelo desencadeamento ou piora de problemas como solidão, ansiedade, depressão, drogadição, estresse pós-traumático, entre outros (Gundim et al., 2021). O elevado tempo de afastamento do ambiente e horário acadêmico acentua os impactos do distanciamento na saúde física e mental desses estudantes (Islam et al., 2020).

Ademais, notícias como o aumento de casos confirmados, o longo tempo em quarentena, a mudança para ensino remoto e a diminuição do rendimento nos estudos têm colaborado sobremaneira para o sofrimento mental dos universitários (Maia \& Dias, 2020).

O ensino remoto tem sido uma das ferramentas fundamentais para continuação da carga horária universitária. Entretanto, sua implantação foi complexa e problemática em muitos cenários, e é desgastante para muitos estudantes, acentuando os estresses físico e mental (Islam et al., 2020).

Faz-se necessário apurar o impacto da pandemia na saúde mental dos estudantes do ensino superior, uma vez que têm crescido os relatos de aumento de alterações sentimentais e problemas mentais. Diante disso, o presente estudo tem por objetivo demonstrar as estatísticas relacionadas aos impactos da pandemia na saúde mental dessa população.

\section{Metodologia}

O presente artigo é de natureza bibliográfica na modalidade revisão integrativa da literatura. Uma revisão integrativa consiste em um estudo que fornece compreensão abrangente de um determinado tema. Tendo como objetivo desenvolver um tema proposto em quatro etapas: elaborar a pergunta principal, buscar em dados literários, apresentar os resultados e discuti-los. (Hopia, Latvala, Liimatainen, 2016).

A pergunta que norteou a pesquisa foi: Qual foi o impacto da pandemia do COVID-19 na saúde mental dos estudantes de ensino superior? Para responder à questão norteadora, as fontes foram obtidas através dos seguintes bancos de dados on-line: Literatura Latino-Americana e do Caribe em Ciências da Saúde (LILACS), Scientific Electronic Library Online (SciELO), e 
Pubmed via Medline. A busca foi realizada com palavras-chave indexadas nos Descritores em Ciências da Saúde (DECs), em inglês: Students, Student Health, COVID-19, Mental health, em espanhol: Estudiantes, COVID-19, Salud del Estudiante, Salud Mental, em francês: Étudiants, COVID-19, Santé des Élèves, Santé mentale e em português: Estudantes, COVID-19, Saúde dos Estudantes e Saúde Mental realizando o cruzamento entre os descritores através do operador booleano AND.

Em relação aos critérios de elegibilidade foram definidos como inclusão: texto completo em Português, Inglês, Espanhol, Francês que abordassem o tema relacionando a saúde mental dos estudantes e a pandemia do COVID-19 nos anos de 2020 e 2021. Após leitura, foram excluídos: teses, dissertações, editorial, carta ao editor, opiniões e revisões de literatura.

Inicialmente foram encontrados 202 artigos através das pesquisas nas bases de dados eletrônicas. Após análise inicial das fontes, foram identificados 104 artigos repetidos. Após leitura dos títulos e resumos, foram excluídos 88 artigos com temática em desacordo aos interesses da pesquisa. A amostra final contou com apenas 10 artigos com para a inclusão definitiva neste estudo.

Quanto aos aspectos éticos, o presente estudo fez uso de dados secundários respeitando a autoria com citações, conforme lei n 9.610, de 19 de Fevereiro de 1998.

\section{Resultados}

O fluxograma das etapas de seleção dos artigos, assim como os resultados da estratégia de busca são apresentados na Figura 1. A seleção se deu de forma criteriosa e sistemática e os passos referentes à seleção e exclusão dos estudos estão dispostos no fluxograma desenvolvido pelo The PRISMA Statement (Figura 1).

Figura 1: Seleção dos artigos por grupos de descritores nas bases de dados.

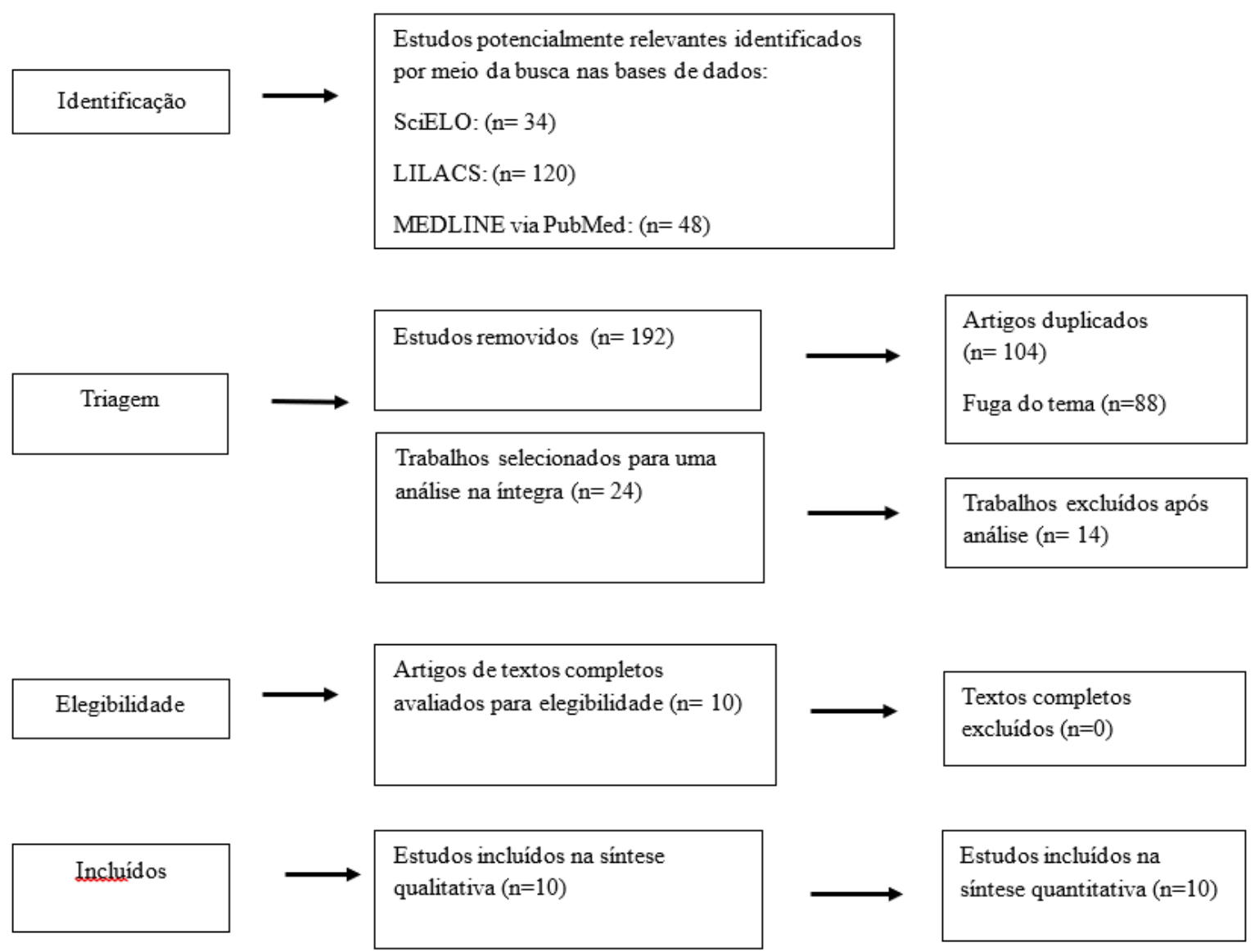

Fonte: Autores. 
Dentre os artigos selecionados, $20 \%$ dos textos são da língua portuguesa, $20 \%$ na língua espanhola, e por fim, $60 \%$ na língua inglesa. Vale ressaltar que, nas bases de dados selecionadas, não foram encontrados artigos na língua francesa. Ao tratar dos anos de publicação, os estudos foram limitados aos anos de 2020 e 2021, sendo $50 \%$ publicados no ano de 2020 e $50 \%$ no ano de 2021. Conforme exposto na Quadro 1.

Quadro 1: Descrição dos artigos selecionados em ordem crescente. (ISME - Impacto na saúde mental dos estudantes).

\begin{tabular}{|c|c|c|c|c|}
\hline Autores & Título & Periódico & Ano/código & Principais resultados \\
\hline $\begin{array}{l}\text { LASHE } \\
\text { RAS, I, } \\
\text { et al. }\end{array}$ & $\begin{array}{l}\text { Prevalence of Anxiety in } \\
\text { Medical Students during the } \\
\text { COVID-19 Pandemic: A Rapid } \\
\text { Systematic Review with Meta- } \\
\text { Analysis }\end{array}$ & $\begin{array}{l}\text { International } \\
\text { Journal of } \\
\text { Environmental } \\
\text { Research and } \\
\text { Public Health }\end{array}$ & $\begin{array}{c}2020 \\
\text { (ISME 1) }\end{array}$ & $\begin{array}{l}\text { A prevalência de ansiedade em estudantes de medicina é } \\
\text { semelhante à anterior à pandemia, mas se correlaciona } \\
\text { com vários estressores específicos relacionados ao } \\
\text { COVID. }\end{array}$ \\
\hline $\begin{array}{l}\text { ISLAM. } \\
\text {. S, et } \\
\text { al. }\end{array}$ & $\begin{array}{l}\text { Psychological responses during } \\
\text { the COVID-19 } \text { outbreak among } \\
\text { university } \quad \text { students in } \\
\text { Bangladesh }\end{array}$ & $\begin{array}{l}\text { Public Library } \\
\text { of Science } \\
\text { (PLoS) }\end{array}$ & $\begin{array}{c}2020 \\
\text { (ISME 2) }\end{array}$ & $\begin{array}{l}\text { Análise dos principais efeitos causados aos estudantes } \\
\text { universitários de Bangladesh durante a pandemia da } \\
\text { COVID-19. }\end{array}$ \\
\hline $\begin{array}{l}\text { MENA, } \\
\text { C, et al. }\end{array}$ & $\begin{array}{l}\text { Intervención psicoterapéutica } \\
\text { breve en estudiantes caribeños } \\
\text { de Medicina con reacciones } \\
\text { psicológicas por impacto ante la } \\
\text { COVID-19 }\end{array}$ & $\begin{array}{l}\text { Medisan: } \\
\text { Revista } \\
\text { Médica de } \\
\text { Santiago de } \\
\text { Cuba }\end{array}$ & $\begin{array}{c}2020 \\
\text { (ISME 3) }\end{array}$ & $\begin{array}{l}\text { Houve prevalência do sexo feminino entre as idades de } \\
\text { de } 20 \text { a } 30 \text { anos. Após a intervenção houve redução tácita } \\
\text { dos níveis de ansiedade e sintomas depressivos, e obteve- } \\
\text { se evolução clínica favorável. }\end{array}$ \\
\hline $\begin{array}{l}\text { ABREU } \\
\text {, MRP, } \\
\text { et al. }\end{array}$ & $\begin{array}{l}\text { Alteraciones psicológicas en } \\
\text { estudiantes de medicina durante } \\
\text { la pesquisa activa de la COVID- } \\
19\end{array}$ & $\begin{array}{l}\text { Medisan: } \\
\text { Revista } \\
\text { Médica de } \\
\text { Santiago de } \\
\quad \text { Cuba }\end{array}$ & $\begin{array}{c}2020 \\
\text { (ISME 4) }\end{array}$ & $\begin{array}{l}\text { Determinar manifestações psicológicas em estudantes de } \\
\text { medicina durante a pesquisa ativa do COVID- } 19 \text {. }\end{array}$ \\
\hline $\begin{array}{l}\text { MAIA, } \\
\text { BR, et } \\
\text { al. }\end{array}$ & $\begin{array}{l}\text { Ansiedade, depressão e estresse } \\
\text { em estudantes universitários: o } \\
\text { impacto da covid-19 }\end{array}$ & $\begin{array}{l}\text { Estudos de } \\
\text { Psicologia } \\
\text { (Campinas) }\end{array}$ & $\begin{array}{c}2020 \\
\text { (ISME 5) }\end{array}$ & $\begin{array}{l}\text { Os estudantes que integraram o estudo no período } \\
\text { pandêmico apresentaram níveis significativamente mais } \\
\text { elevados de depressão, ansiedade e estresse } \\
\text { comparativamente aos que integraram o estudo no } \\
\text { período normal. Os resultados sugerem um impacto } \\
\text { psicológico negativo da pandemia nos estudantes. }\end{array}$ \\
\hline $\begin{array}{l}\text { GARCÍ } \\
\text { A, R, et } \\
\text { al. }\end{array}$ & $\begin{array}{l}\text { Psychological distress, sanitary } \\
\text { measures and health status in } \\
\text { student's university }\end{array}$ & $\begin{array}{c}\text { Nova Scientia: } \\
\text { Revista de } \\
\text { Investigación } \\
\text { de la } \\
\text { Universidad } \\
\text { de LaSalle } \\
\text { Bajio }\end{array}$ & $\begin{array}{c}2021 \\
\text { (ISME 6) }\end{array}$ & $\begin{array}{l}\text { Identificou-se maior risco de apresentar sofrimento } \\
\text { psíquico em mulheres, pós-graduandos, pertencentes às } \\
\text { áreas de saúde e humanidades, apresentando obesidade e } \\
\text { preocupação em serem infectadas, não seguindo medidas } \\
\text { sanitárias e apresentando qualquer desconforto físico. }\end{array}$ \\
\hline $\begin{array}{c}\text { LUO, } \\
\text { W, et al. }\end{array}$ & $\begin{array}{l}\text { Prevalence of depressive } \\
\text { symptoms among Chinese } \\
\text { university students amid the } \\
\text { COVID-19 pandemic: a } \\
\text { systematic review and meta- } \\
\text { analysis }\end{array}$ & $\begin{array}{l}\text { Epidemiology } \\
\text { and } \\
\text { Psychiatric } \\
\text { Sciences }\end{array}$ & $\begin{array}{c}2021 \\
\text { (ISME 7) }\end{array}$ & $\begin{array}{l}\text { A prevalência agrupada de sintomas depressivos } \\
\text { significativamente maior no sexo feminino do que no } \\
\text { sexo masculino, em estudantes que tiveram conhecidos } \\
\text { ou parentes infectados com COVID-19 do que naqueles } \\
\text { que não o tiveram. }\end{array}$ \\
\hline
\end{tabular}




\begin{tabular}{|c|c|c|c|c|}
\hline $\begin{array}{l}\text { DENG, } \\
\mathrm{J} \text {, et al. }\end{array}$ & $\begin{array}{l}\text { The prevalence of depressive } \\
\text { symptoms, anxiety symptoms } \\
\text { and sleep disturbance in higher } \\
\text { education students during the } \\
\text { COVID-19 pandemic: a } \\
\text { systematic review and meta- } \\
\text { analysis }\end{array}$ & $\begin{array}{l}\text { Psychiatry } \\
\text { Research }\end{array}$ & $\begin{array}{c}2021 \\
\text { (ISME 8) }\end{array}$ & $\begin{array}{l}\text { A prevalência de sintomas depressivos e sintomas de } \\
\text { ansiedade sintetizados neste estudo foi maior em } \\
\text { comparação com a prevalência pré-pandemia em } \\
\text { populações semelhantes.O rastreamento e a intervenção } \\
\text { em saúde mental devem ser prioridade para as } \\
\text { universidades durante a pandemia. }\end{array}$ \\
\hline $\begin{array}{l}\text { TEIXEI } \\
\text { RA, } \\
\text { LAC, et } \\
\quad \text { al. }\end{array}$ & $\begin{array}{l}\text { Saúde mental dos estudantes de } \\
\text { Medicina do Brasil durante a } \\
\text { pandemia da coronavírus disease } \\
2019\end{array}$ & $\begin{array}{c}\text { Jornal } \\
\text { Brasileiro de } \\
\text { Psiquiatria }\end{array}$ & $\begin{array}{c}2021 \\
\text { (ISME 9) }\end{array}$ & $\begin{array}{l}\text { O estudo demonstrou que os indícios de sofrimento } \\
\text { psíquico estão elevados entre estudantes de Medicina } \\
\text { durante a pandemia da COVID-19. Além disso, também } \\
\text { foi possível concluir que há fatores protetores para o } \\
\text { adoecimento mental. }\end{array}$ \\
\hline $\begin{array}{l}\text { SANTA } \\
\text { BÁRBA } \\
\text { RA, J, } \\
\text { et al. }\end{array}$ & $\begin{array}{l}\text { Prevalence of anxiety in the } \\
\text { COVID-19 pandemic: an } \\
\text { updated meta-analysis of } \\
\text { community-based studies }\end{array}$ & $\begin{array}{l}\text { Progress in } \\
\text { Neuro- } \\
\text { Psychopharma } \\
\text { cology and } \\
\text { Biological } \\
\text { Psychiatry }\end{array}$ & $\begin{array}{c}2021 \\
\text { (ISME 10) }\end{array}$ & $\begin{array}{l}\text { Fatores de risco consistentemente relatados para o } \\
\text { desenvolvimento da ansiedade incluíram fase inicial ou } \\
\text { de pico do surto, sexo feminino, idade mais jovem, } \\
\text { casamento, isolamento social, desemprego e status } \\
\text { estudantil, dificuldades financeiras, baixo nível } \\
\text { educacional, conhecimento insuficiente do COVID-19. }\end{array}$ \\
\hline
\end{tabular}

Fonte: Alves IFRD, et. al. (2021).

Tendo em vista os artigos analisados pelo presente estudo, foi possível evidenciar o aumento de manifestações psicológicas negativas nos estudantes de ensino superior durante a pandemia da COVID-19, sendo relatado esse aumento em $100 \%$ dos estudos compilados para fomentar a pesquisa realizada.

\section{Discussão}

Atualmente, os estudantes universitários sofrem os impactos na saúde mental em decorrência de diversas fontes estressoras fruto da pandemia, como: afastamento social, a falta de informações claras dadas pelas autoridades área de saúde e sanitárias, prejuízos financeiros, mudanças repentinas na rotina, perdas de produtividade nos estudos, preocupações com a saúde, entre outros (Marroquín, Vine \& Morgan, 2020, Lasheras et al., 2020).

Apesar das medidas de isolamento serem extremamente necessárias para evitar a disseminação da COVID-19, esses padrões acarretam aumento dos sentimentos de solidão, desesperança, medo e frustrações. (ISLAM et al., 2020) Anteriormente a pandemia, a ansiedade e a depressão já eram consideradas as doenças mentais mais prevalentes no mundo, mas no quadro vigente, ficou cada vez mais comum emoções e vivências negativas, ocasionando dificuldades de curto prazo, podendo acarretar problemas mentais. Problemas esses que podem ocasionar, entre outras coisas, transtorno de sono, mudanças alimentares e experiências viciantes. Além disso, notícias relacionadas ao número de doentes e mortos, as "fake news" divulgadas amplamente nos meios de comunicação e a infodemia acerca da pandemia da COVID-19 apresentam relatos de dificuldades em problemas emocionais. (Luo, Zhong \& Chiu, 2021, Castaldelli-Maia et al., 2021).

Ainda nesse sentido, um estudo realizado por Deng et al., (2021) incluiu 89 estudos para a sua amostra final em uma revisão sistemática onde os distúrbios de depressão foram encontrados em $34 \%$, os de ansiedade em $32 \%$ e os distúrbios de sono $33 \%$ quando comparados ao período pré pandemico. Outros fatores contribuíram para o aumento dos sintomas de ansiedade e depressão nos universitários, entre eles a redução da carga horária em alguns cursos (principalmente na área da saúde) e o distanciamento social também contribuíram cada vez mais para os riscos de desenvolvimento e agravamento de doenças mentais nos universitários (Ramírez et al., 2021, Santabárbara et al., 2020, Maia \& Dias, 2020). 
Embora sejam medidas e restrições estabelecidas pelo governo e demais autoridades para o controle da infeção, esses parâmetros repentinos foram muito significativos na rotina dos estudantes, privando-os de aconselhamento, convivência, desenvolvimento pessoal e consolidação de relações formais e informais. Desse modo, levando também a maior possibilidade de desenvolvimento das doenças mencionadas (Castaldelli-Maia et al., 2021, Deng et al., 2021).

Durante a pandemia, escolas, Instituições de Ensino Superior (IES), permaneceram fechadas, sendo essa uma das medidas de contenção da COVID-19 . Consoante a isso, houve a implementação do ensino remoto virtual para suprir as demandas educacionais vigentes (Islam et al., 2020, Teixeira, et al., 2021).

Um artigo publicado no jornal brasileiro de psiquiatria realizou um estudo transversal observando 656 estudantes de ensino superior, cursando medicina, utilizando questionários, e quando observado os resultados $62 \%$ dos pacientes apresentavam algum sofrimento psíquico. (Teixeira et al., 2021).

Contudo, é perceptível a dificuldade dos alunos em se adaptar à nova realidade tecnológica de ensino, manifestando indícios de déficit de atenção, fadiga mental devido às novas demandas, aflição com o acúmulo de conteúdo, indicaram relação com o adoecimento mental. A prevalência desse adoecimento durante a graduação pode levar a efeitos danosos e irreversíveis à saúde (Gutiérrez et al., 2021).

Mesmo que os efeitos da COVID-19 de ordem psicológica atinjam os estudantes de graduação de todas as áreas, devese ressaltar em especial àqueles da área da saúde devido a maior exposição ao contágio e probabilidade de desenvolver reações de pânico, angústia, ansiedade, depressão, irritabilidade, agressividade, transtorno de estresse pós-traumático e síndrome de burnout, possibilitando repercutir sobre si mesmo e sua família (Cobián et al., 2020).

Dessa forma, os cuidados de saúde da população impõe uma sobrecarga emocional não somente aos profissionais da saúde, mas também aos estudantes desse âmbito (Reshetnikov, 2021, Perez et al., 2020).

\section{Conclusão}

Constatou-se que a maioria dos estudantes do ensino superior apresentam relatos de doenças mentais que podem estar relacionadas à falta de interação social. Desse modo, faz-se necessário que o setor de saúde providencie medidas de prevenção contra a vulnerabilidade de doenças mentais na pandemia e que dê prioridades para esses estudantes das IES. Perante o exposto, este estudo buscou contribuir para reconhecimento de dados e para em breve sirva em prol as organizações de saúde com providências e soluções para a diminuição de casos de estudantes universitários com problemas mentais.

Como perspectivas futuras, recomenda-se, que sejam realizados outros estudos que relacionem a saúde mental dos estudantes e a pandemia do COVID-19, estudos de ensaio clínico com metodologia criteriosa, assim como estudos sem limitação linguística, que pode ter de certa forma influenciado nos resultados encontrados.

\section{Referências}

Castaldelli-maia, J. M., et al. (2021) Investigating the effect of national government physical distancing measures on depression and anxiety during the COVID19 pandemic through meta-analysis and meta-regression. Psychological medicine, 51(6):881-893 0.1017/S0033291721000933.

Castro V. R. (2017) Reflexões Sobre A Saúde Mental Do Estudante Universitário: Estudo Empírico Com Estudantes De Uma Instituição Pública De Ensino Superior. Ver, Gestão em Foco 9: 380-401. 10.1590/1413-81232014193.11042012

Cobián M. A. E. et al., (2020) Intervención psicoterapéutica breve en estudiantes caribeños de Medicina con reacciones psicológicas por impacto ante la COVID-19. Medisan, 24(5):823-835

Deng J, et al., (2021) The prevalence of depressive symptoms, anxiety symptoms and sleep disturbance in higher education students during the COVID-19 pandemic: A systematic review and meta-analysis. Psychiatry Research, 301: e:113863 10.1016/j.psychres.2021.113863

Gama C. A. P. \& Campos R. T. O \& Ferrer, A. L., (2014) Saúde mental e vulnerabilidade social: a direção do tratamento. Rev. latinoam. psicopatol. fundam, 17(1): 69-84 10.1590/S1415-47142014000100006 
Gundim V. A. et al., (2021) Saúde mental de estudantes universitários durante a pandemia de COVID-19. Rev baiana enferm;35: e37293: 1-14 $10.18471 /$ rbe.v35.37293

Gutiérrez G. R. et al., (2021) Psychological distress, sanitary measures and health status in student's universitary. Nova Scientia; 35: e37293. 10.21640/ns.v13ie.2602

Hopia H, et al., (2016) Reviewing the methodology of an integrative review. Scandinavian Journal Of Caring Sciences; 30(4): 662-669. 10.1111/scs.12327

Islam MS, et al., (2020) Psychological responses during the COVID-19 outbreak among university students in Bangladesh. PLoS One, 15(12):1-15; DOI:10.1371 / journal.pone.0245083

Lasheras I, et al., (2020) Prevalence of Anxiety in Medical Students during the COVID-19 Pandemic: A Rapid Systematic Review with Meta-Analysis. International journal of environmental research and public health; 17(18): 1-12. 10.3390 / ijerph17186603.

Luo W. et al., (2021) Prevalence of depressive symptoms among Chinese university students amid the COVID-19 pandemic: a systematic review and metaanalysis. Epidemiol Psychiatr Sci, 30; e:31:1-21. 10.1017 / S2045796021000202

Maia B. R. \& Dias P. C., (2020) Ansiedade, depressão e estresse em estudantes universitários: o impacto da COVID-19. Estudos de psicologia, 37; e200067, 18 10.1590/1982-0275202037e200067

Marroquín B. et al., (2020) Mental health during the COVID-19 pandemic: effects of stay-at-home policies, social distancing behavior, and social resources. Psychiatry Research, 293: 113419. 10.1016 / j.psychres.2020.113419.

Pereira A. S. et al., (2018). Metodologia da pesquisa científica. UFSM.

Perez A. M. R. et al., (2020) Alteraciones psicológicas en estudiantes de medicina durante la pesquisa activa de la COVID-19. Medisan,; 24(4): 537-548.

Ramírez F. B., et al., (2021) Repercusiones de la pandemia de la COVID-19 en la salud mental de la población general. Atención Primaria, 53(7): 102143. 10.1016/j.aprim.2021.102143

Reshetnikov V, et al., (2021) Indoor Environmental Quality in Dwellings and Lifestyle Behaviors during the COVID-19 Pandemic: russian perspective. International Journal Of Environmental Research And Public Health, 18(11): 5975. 10.3390/ijerph18115975

Santabárbara J, et al., (2020) Prevalence of anxiety in the COVID-19 pandemic: An updated meta-analysis of community-based studies. Progress in neuropsychopharmacology \& biological psychiatry; 13;109:110207 10.1016/ j.pnpbp.2020.110207

Santos J. V. B. S. \& Monteiro, J. C. S. (2020) Educação e Covid-19: As tecnologias digitais mediando a aprendizagem em tempos de pandemia. Revista Encantar. 2: 01-15 10.46375/encantar.v2.0011

Teixeira, L.A.C. et al., (2021) Saúde mental dos estudantes de Medicina do Brasil durante a pandemia da coronavirus disease 2019. J Bras Psiquiatr.; 70 (1): 21-29 10.1590/0047-2085000000315 